Review

\title{
Epstein barr virus encodes miRNAs to assist host immune escape
}

\author{
Weiming $\mathrm{Li}^{1}$, Cong $\mathrm{He}^{1}$, Jiayi Wu² , Dazhi Yang ${ }^{1,2 \bowtie}$, Weihong $\mathrm{Yi}^{1,2}{ }^{\bowtie}$ \\ 1. Department of orthopedics, Union Shenzhen Hospital, Huazhong University of Science and Technology, Shenzhen, Guangdong, China \\ 2. Department of orthopedics, The 6th Affiliated Hospital of Shenzhen University Health Science Center, Shenzhen, Guangdong, China \\ $\bowtie$ Corresponding authors: Weihong Yi, Email: szywh@email.szu.edu.cn; Dazhi Yang, Email: dazhiyang@email.szu.edu.cn. Tel: +86-755-86671903
}

(1) The author(s). This is an open access article distributed under the terms of the Creative Commons Attribution License (https://creativecommons.org/licenses/by/4.0/). See http://ivyspring.com/terms for full terms and conditions.

Received: 2019.11.26; Accepted: 2020.01.10; Published: 2020.02.03

\begin{abstract}
Epstein-barr virus (EBV) is a definite tumorigenic virus, which can form life-long latency in the host, which is difficult to be recognized and completely eliminated by the immune system. It is closely related to the occurrence and development of nasopharyngeal cancer, gastric cancer and various types of lymphoma. At present, a total of 44 Epstein-barr virus-encoded microRNAs (EBV miRNAs) have been found. In response to the immune system of the body, EBV miRNAs can inhibit the expression and presentation of viral antigens, inhibit immune activation and immunotoxicity, assisting host cells to escape from immunity, and providing conditions for further immortalized tumorigenesis of the host cells.
\end{abstract}

Key words: EBV miRNAs, immune escape, tumor

\section{Introduction}

EBV is a human herpesvirus type IV, belonging to the human herpesvirus gamma subfamily [1]. It was first discovered and named by Anthony Epstein and Yvonne Barr in 1964 [2]. EBV is a harmful pathogen and is closely related to the occurrence of nasopharyngeal carcinoma and Burkitt's lymphoma. More and more evidence indicates that EBV is also the cause of Hodgkin's lymphoma, B and T/NK non Hodgkin lymphomas and gastric cancer [3-6]. Currently, it is known that EBV has caused at least 200,000 new cancer patients every year [7].

EBV infection is mainly transmitted through saliva, but can also be infected through blood transfusion, and the infection rate in adults is as high as $90 \%$ [8]. The first infection of EBV occurs mainly in the epithelial cells of the pharynx of the population, followed by infection of B lymphocytes. As B lymphocytes carrying EBV enter the blood circulatory system, systemic EBV infection can occur $[9,10]$. In the face of host immune response and immunotoxicity, EBV can derive corresponding strategies to assist the host cells to immune escape, and establish a lifetime of latency, which is difficult to be completely eliminated by the body's immune system [11].

After EBV infects the host, EBV antigen is encoded and expressed. Currently, specific antigens including Epstein-barr virus nuclear antigen (EBNA), Epstein-barr virus capsid antigen (EBVCA), Epsteinbarr virus early antigen (EA), Epatein-barr virus membrane antigen (MA), etc. [12]. EBNA is a latent gene of EBV, in addition to latent genes such as EBERs, BARTs, and EBNA-LP. According to the latent genes expressed by EBV in host cells, hosts can be classified into Four latency (type 0, type I, type II, and type III) $[13,14]$. The EBV of type 0 latency expresses only one latent gene in the host cell is EBV-encoded RNAs (EBERs). For example, infected dormant memory B cells belong to type 0 latency [15]. The EBV of type I latency expresses three latent genes in the host cell, EBNA1, EBERs, and BARTs (BamHI-A rightward transcripts). Such as burkitt's lymphoma and plasmablastic lymphoma, which belong to type I $[16,17]$. The latent genes of EBV expression in the host with type II latency include four latent genes: EBNA1, EBERs, BARTs, LPMs (latent membrane protein). For example, hodgkin's lymphoma, nasopharyngeal carcinoma, diffuse large B-cell lymphoma, chronic 
lymphocytic leukemia and EBV-associated gastric cancer are type II latency [18-21]. The hosts with type III latency express eight latent genes, such as EBERs, EBNA1, EBNA-LP (EBNA-leader protein), EBNA2, EBNA3A, EBNA3B, EBNA3C, LPMs. For example, immunoblastic lymphoma, diffuse large $B$ of the elderly and pyothorax associated lymphoma belong to type III $[19,22]$.

Interestingly, EBV is usually a type I latency in PBL patients, expressing EBNA1, EBERs, and BARTs $[16,23]$. However, in a recent study of miRNA sequencing in PBL, it was found that EBV also had type II latency in PBL patients and expressed LMP1, suggesting that the latency state of EBV in tumor is not constant, and the change of latency may be related to immune regulation of EBV[24]. PBL patients are often associated with HIV and EBV infection [25, 26]. HIV infection has long been considered as the main cause of PBL, while EBV infection has not received much attention. Rodrigues-Fernandes, C.I., et al. made a clinicopathological analysis of 159 cases of
PBL, and found that EBV positive status was one of the independent prognostic determinants of a poor prognosis through a multivariate Cox regression model [27]. In addition, Gravelle, P and Laurent, C, et al. found that compared with EBV negative PBL, there were abundant leukocyte infiltration and $\mathrm{T}$ cell activation signals in EBV positive PBL tissue, high expression of PD-1/PD-L1 immune checkpoint and other immune escape related proteins, and positive correlation with host immunosuppression, suggesting EBV may play a more important role in immune escape $[16,28]$. In the study of miRNA sequencing in PBL also found that the expression of tumor suppressor gene PTEN, PBX2 and metabolic controller PPARGC1A, PLIN2/adipophilin was regulated by BART-19-5p [24]. These studies suggest that EBV plays an important immunomodulatory role in the development of $\mathrm{PBL}$, during which the latency status of EBV may change, and EBV-miRNAs may be involved in different regulatory roles.

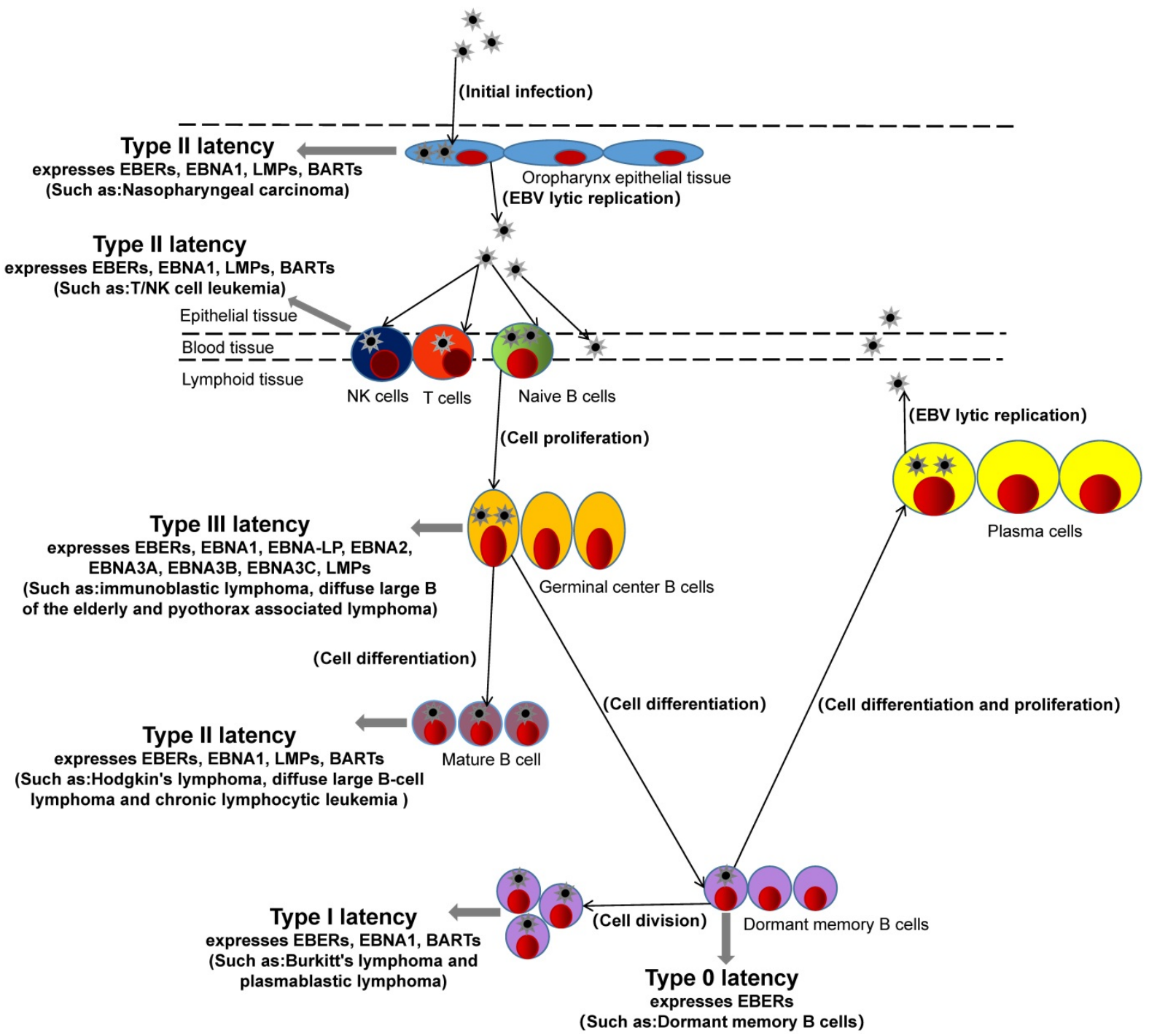

Figure 1. The formation of EBV latent infection 
EBV not only has different types of latency in different hosts and different expressed latent genes but also has different expressions of miRNAs encoding itself in different host tissues with types of latency. For example, EBV-miR-BARTs are widely expressed in the four latency types and lytic replication of EBV in host cells, while the expression of EBV-miR-BHRF1s depends on the type of virus latency and is generally expressed in III latency and lytic replication of host cells $[29,30]$. In addition, the expression of EBV-miR-BHRF1s in lymphoma is very high, while the expression in nasopharyngeal and epithelial tumors is absent [19]. EBV-miRBARTs are expressed in both lymphoma and epithelial tumors. These studies suggest that EBV-miR-BARTs and EBV-miR-BHRH1s play different regulatory roles in different tumors and different latency.

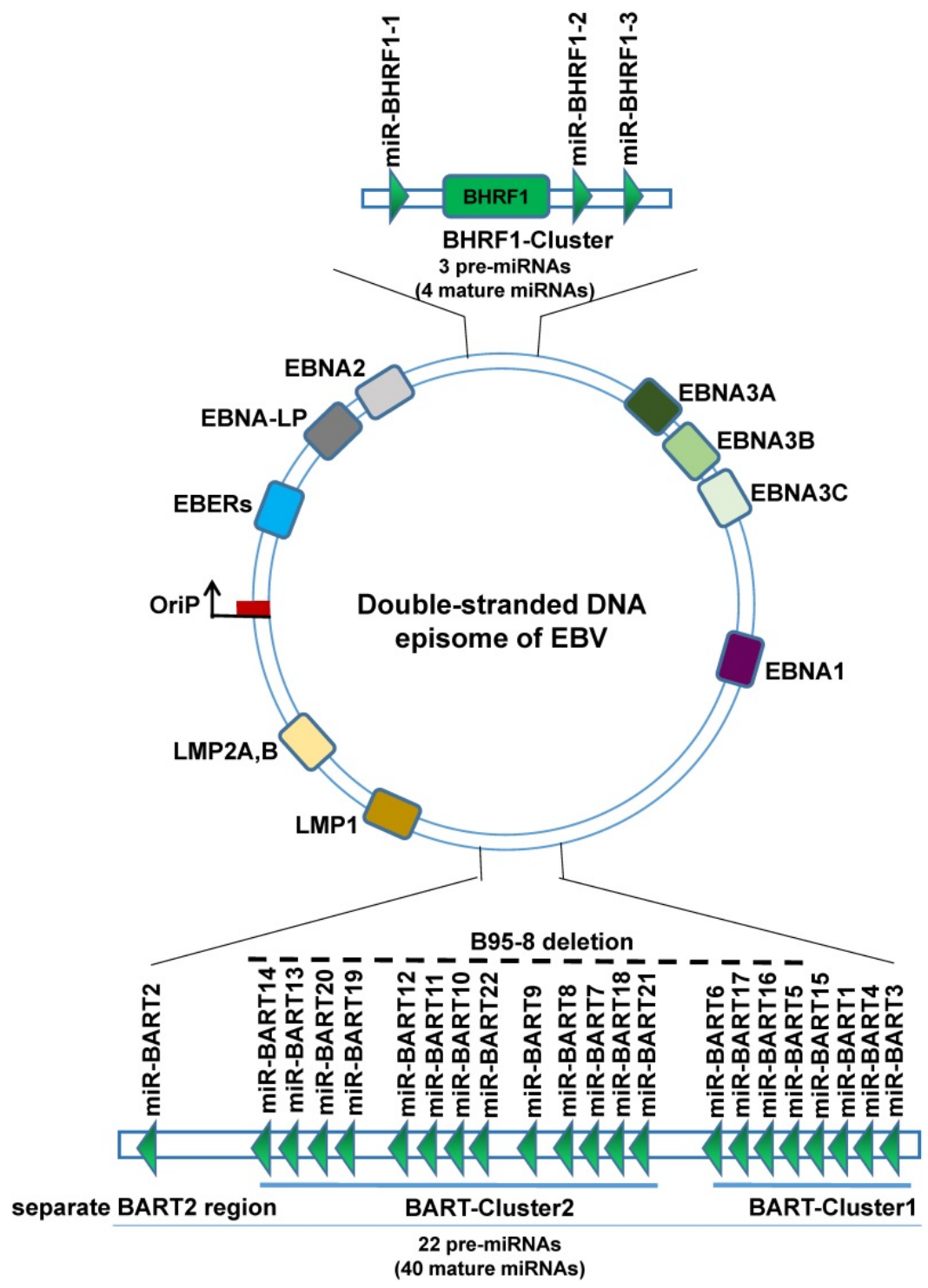

Figure 2. Schematic representation of the genomic locations of EBV-encoded miRNAs. The relative positions of EBV latent and EBV encodes 25 pre-miRNAs genes on the genome are indicated.

\section{EBV miRNAs}

EBV is the first virus discovered by humans to express miRNAs. EBV miRNAs are non-coding small RNA composed of 18-24 nucleotides. So far, a total of $25 \mathrm{EBV}$ miRNAs precursors have been found, forming 44 mature EBV-miRNAs, which are transcribed from the two precursors to the two regions of the EBV genome, namely the BART region and the BHRF1 region, wherein the BART region has two miRNA cluster regions (BART-Cluster 1, BART-Cluster 2) and the separate BART2 region, can transcribe 22 miRNAs precursors to form 40 mature miRNAs. The miRNAs BART-Cluster 1 is located between the BART gene exon 1 and exon $1 B$ and encodes 8 miRNA precursors (EBV-miR-BART1, EBV-miR-BART3 6, and EBVmiR-BART15 17). BART-Cluster 2 is located between exon $1 \mathrm{~B}$ and exon 3 of the BART gene and encodes 13 miRNA precursors (EBV-miR-BART7 14 and EBV-miR-BART18 22). While the BART2 region is located between exon 4 and exon 5, encodes only one precursor (EBV-miR-BART2). The BHRF1-Cluster region transcribes three miRNA precursors (EBV-miR-BHRF1$1 \sim 3)$ to form four mature EBV-miR-BHRF1s. EBV miRNAs are named EBV-miR-BART1 22 and EBV-miR-BHRF1-1 3 according to the transcription region of their genome and the order in which they were found [31-33]. Interestingly, not all EBV strains have complete BART region. The B95-8 laboratory strain of EBV bears a deletion that removes most of the BART region, can immortalize primary $B$ cells in culture, suggesting that BARTs are dispensable for transforming B cells [34].

In a typical miRNA organism, one chain is selective processing into mature miRNA, while the other strand is rapidly degraded, but EBV miRNAs during processing are not all so, most of the EBV miRNAs hairpin structure function related to the two chains are processed into mature miRNA, to distinguish the two miRNAs, according to the $3^{\prime}$ or $5^{\prime}$ end of the miRNA in the hairpin structure named suffix $-3 p$ or $-5 p[35,36]$. Therefore, 25 precursors eventually form 44 mature EBV miRNAs.

After EBV infection of host cells, these EBV miRNAs form incomplete complementary pairing with the 3'-untranslated region (3'UTR) of the 
virus's own genes or host gene mRNAs, resulting in inhibition of gene translation or the degradation of targeted mRNAs [37]. In recent years, many studies have shown that EBV miRNAs can inhibit the expression and presentation of viral antigens by inhibiting the expression of viral genes and host cell genes, inhibiting the activation and cytotoxicity of immune cells, and achieving long-term latency, immune escape, and promotion of host cells immortalization and tumor development [38], which is briefly reviewed below.

\section{Antigen expression}

EBV infected host cell for the first time, to remove the EBV infection, host cells will present the EBV antigens to the cell surface, so that through cell immune clearance of the virus in the cell. In order to evade the body's immune system to kill, EBV miRNAs can regulate host cell EBV antigen expression, to present a lower amount of antigen, and difficult to be immune cells to recognize, to escape immune surveillance at an early stage, create conditions for the latency of EBV [39]. For example, LMP2A is EBV encoded with high immunogenicity of virus antigen, which can be CD4+ and CD8+ T cells specificity recognition [40]. But Lung, R.W., et al. found that in $\mathrm{EBV}+$ nasopharyngeal cells, EBV-miR-BART22 targets the 3'UTR of LMP2A gene mRNA, inhibits the expression of LMP2A, weakens the host's immune response, and makes EBV+ nasopharyngeal cells become a blind area for immune surveillance and immune escape [41].

LMP1, as an EBV-encoded viral antigen, is a kind of oncogenic gene and participates in the activation of signaling pathways such as NF-kB, JNK-p38/SAPK, Ras-MAPK, PI3k-Akt and JAK-STAT, which can promote the occurrence of EMT and make nasopharyngeal carcinoma has a higher invasion and metastasis ability, which is considered to be an important factor in the development of nasopharyngeal carcinoma [42]. Studies have found that EBVmiR-BART9, EBV-miR-BART16 and EBV-miRBART17-5p can both target the 3'UTR of LMP1 mRNA and negatively regulate the expression of LMP1. Although such negative regulation of LMP1 can reduce the invasion and metastasis of tumor cells to a certain extent, it also greatly weakens the host's immune recognition and response, and evade immune surveillance [43-45]. And Lo, A.K., et al. found that very small amounts of LMP1 (below the detection threshold), enough to activate the NF-kB signaling pathways, play a role in promoting the cancer. While this small amount of LMP1, compared with the sensitivity of memory $\mathrm{T}$ cells to the LMP1 epitope, cannot activate memory $\mathrm{T}$ cells of the immune system. This suggests that EBV miRNAs may, by fine-regulating the expression level of LMP1, promote host immortalization of tumors while avoiding immune surveillance and promote the development of tumors [46].

EBNA1 is the first discovered EBV antigen, expressed in host cells of various latency types at the proliferative stage. Although EBNA1 can be T cells target recognition, the host of EBNA1 almost not be $\mathrm{MCH}$ I presented to the surface of cells. Studies also found that EBV miRNAs can inhibit EBNA1 expression, which may be EBV miRNAs a means to assist the host to escape immune surveillance [47]. In addition, EBV miR-BART6-5p was found to inhibit EBNA2 expression [48], and EBV-miR-BHRF1s could inhibit EBNA2 co-activated antigen EBNA-LP expression [49], suggesting that EBNAs and EBNA-LP could be regulated by EBV miRNAs to avoid immune surveillance and maintain EBV latency.

\section{Antigen presentation}

After EBV infection of the host, to avoid exposure by host cells, evade immune system recognition, EBV miRNA not only participates in the regulation of EBV antigen expression but also regulates the presentation of EBV antigen during the process of EBV antigen transport to the host surface. It can avoid large amounts of antigen exposure and be recognized and attacked by immune cells, helping the host immune escape. After EBV infection, the antigen peptide segment formed by EBV antigen in the cytoplasm after treatment by proteasome enzyme is transported to the endoplasmic reticulum cavity by transporter associated with antigen processing (TAP), and then combined with major histocompatibility complex I (MHC I) to further present to the cell surface, then CD80+ Cytotoxic lymphocyte (CTL) was activated for specific identification of cull [50]. The TAP of is located on the endoplasmic reticulum and cis-Golgi membrane in this process. TAP is composed of two subunits TAP1 and TAP2, and the loss of any subunit will make TAP ineffective and lead to the significant down-regulation of MHC I on the cell surface [50]. Lin, T.C., et al. found that EBV miR-BHRF1-3 and EBV miR-BART17 directly target the 3'UTR of TAP2 mRNA, weaken EBV antigen presentation and MHC I cell surface expression, thereby inhibiting CD80+CTL cells' role in endogenous antigen recognition and killing host cells, resulting in immune escape [51].

When the host EBV antigen is presented to the cell surface, it not only activates the CTL to kill the cells but also activates antigen-presenting cells (APC) to uptake and process the antigen. In this process, the exogenous EBV antigen passes through the lympho- 
cyte antigen (Lymphocyte antigen, LY) transports from the cell surface to lysosomes or endosomes, degraded to antigenic peptides by lysosomal enzymes or endosomal enzymes, and combined with major histocompatibility complex II (MHC II) [52]. Lymphocyte antigen 75 (LY75) plays an important role in the transfer of exogenous EBV antigen from the cell surface to lysosomes or endosomes and activates CD4+ helper T cell (Th cells) and CD8+ CTL Cells [53]. Skalsky, R.L., et al. found that EBV miR-BART1-5p can directly target LY75. Overexpression of EBV miR-BART1-5p in EBV-associated tumor cells can inhibit the expression of LY75, and promote tumor cells to get rid of the recognition and monitoring of CD4+ Th cells and CD80+ CTL cells [54].

After EBV antigen is transported from the cell surface to the lysosome or endosomes, it needs to be degraded into an antigenic peptide by the lysosomal enzyme or endosomal enzyme, and then the processed antigen is presented to $\mathrm{CD} 4+\mathrm{Th}$ cell to activate the immune response by combining with MHC II [55]. EBV miRNAs can regulate the expression of various lysosomal enzymes, inhibit the degradation of antigens, block the binding of antigens to MHC II, and attenuate the activation of CD4+Th cells. It was found that the 3'UTR of mRNA of the three lysosomal enzymes IFI30, LGMN and CTSB in APC cells were targeted by EBV miR-BART1, EBV miR-BART2, and EBV miR-BHRF1-2, thereby inhibiting antigen processing, down-regulating the binding and presentation of EBV antigen peptide fragment and MHC II, and weakening the activation of CD4+Th cells, suppressing the immune response and assisting EBV immune escape [56]. In addition, in addition to directly regulating antigen presentation, EBV miRNAs can indirectly inhibit the expression of costimulatory molecules and MHCs to block antigen presentation, inhibit subsequent immune activation, and evade the monitoring of host immune system [43, 56].

\section{EBV miRNAs regulate the activation of immune cells}

After EBV infects the host, it does not initiate lytic replication immediately but is in a state of persistent latency. When the host's immunity declines, the EBV in the latency state can be activated immediately, forming a recurrent infection, and a large number of synthetic EBVs self-products, including immune antigens such as EBNAs and LMPs, cause host immune responses. First, activate humoral immunity, induce the production of anti-EBNA antibodies, anti-EA antibodies, anti-MA antibodies and anti-VCA antibodies to resist EBV invasion, but that does not completely eliminate the invading virus, especially for latent infection of EBV. Cellular immunity plays an important role in monitoring viral activation and clearance of host cells at this time [57, 58]. In the process of cellular immune response, the two most important roles are lymphocytes and host cells. Studies have found that EBV miRNAs can not only regulate the expression and presentation of EBV antigen in host cells but also regulate the activation and toxicity of immune cells and assist host cells in immune escape in the process of host immune defense.

\section{Direct regulation of immune cell activation}

When the EBV infects the host, the host will produce a ligand-protein MICB, MICB is a ligand for the NKG2D receptor, and the NKG2D receptor is a receptor on the NK cell. Its activation can effectively activate NK cells, by recognizing MICB ligands on the host, and can eliminate infected host cells. Nachmani, D., et al. found that EBV-miR-BART2-5p inhibits the expression of MICB in the host cell by targeting the MICB mRNA on the host cell, reduce the sensitivity of identifying of the host cell by NK cells, block the activation of NK cells, thus protecting the host cell, escape immune recognition and killing of NK cells [59].

When EBV re-infect the host, dormant B cells, driven by the germinal center, can rapidly differentiate into memory B cells. This process is critical for removing the EBV of the intrusion. EBF1 is a transcription factor of B lymphocytes, and is a necessary condition for the development of germinal centers [60]. And EBF1 also targets many B lymphocyte-specific genes, including Paired box 5(PAX5) and B cell receptor (BCR), these two genes are essential for maintaining the function of mature $B$ lymphocyte[61]. Ross, N.et al. found that EBV-miRBART11-5p can binds to the 3'UTR of EBF1 mRNA, inhibits the expression of EBF1, and negatively regulates the proliferation and differentiation of $\mathrm{B}$ lymphocytes, thereby blocking the rapid differentiation and immune activation of $B$ lymphocytes [62].

The rapid differentiation of $B$ lymphocytes in dormancy does not only require germinal center drive, but BCR and PRDM1 are equally important for antigen recognition, antigen-stimulating signal transduction, $\mathrm{B}$ lymphocyte activation and rapid differentiation $[63,64]$. Chen, Y., et al. found that EBV miR-BHRF1-2-5p and EBV miR-BART2-5p in EBV positive $B$ lymphocytes can regulate the $B C R$ pathway, affect the transformation of $B$ cells with latent EBV infection during the Latency and lysis period, inhibit the reactivation and differentiation of $B$ lymphocytes, and assist the immune escape of 
infected host cells [65]. PRDM1 is the main regulator of the terminal differentiation of B lymphocytes and a tumor suppressor gene. $\mathrm{Ma}$, J., et al. have found that PRDM1 expression is very low in EBV+ diffuse large b-cell lymphoma (DLBCL) and Hodgkin's lymphoma, while inhibiting the expression of endogenous EBV miR-BHRF1-2 can significantly improve the expression of PRDM1. Further studies have found that EBV miR-BHRF1-2 can targets and down-regulates PRDM1 expression and inhibits B lymphocyte differentiation and immune activation by targeting the 3'UTR' of PRDM1 mRNA, at the same time promote the $\mathrm{B}$ lymphocyte differentiation and $\mathrm{B}$ lymphocyte immortalized [66].

After EBV infection of lymphocytes, EBV miRNAs can reduce the sensitivity of lymphocyte immune recognition, reduce the cytotoxicity of lymphocytes, protect host cells, and evade immune attack. IPO7 is a nuclear receptor protein and a transcription factor that regulates immune tolerance. The expression level of IPO7 is positively correlated with the cytotoxicity of T lymphocytes [67]. Dolken, L., et al. found that in EBV-positive T lymphocytes, EBV-miR-BART3 targets the 3'UTR of IPO7 mRNA, inhibits the expression of IPO7, reduces the cytotoxicity of T lymphocytes, and assists host cells in immune escape [68]. In addition, Mucosa-associated lymphoid tissue lymphoma transport protein 1 (MALT1) plays an important role in the activation of CD4+ T cells in the immune response [69], while EBV miR-BHRF1-2-5p inhibits the expression of MALT1, thereby inhibiting the activation and cytotoxicity of CD4+ T cells $[70,71]$.

\section{Indirect regulation of immune cell activation}

\section{Interleukin expression and release}

After EBV infection in the host, the expressed EBV antigen is exposed by endogenous and exogenous presentation, recognized by lymphocytes, and activated by the immune response of related lymphocytes to eliminate EBV. Interleukin (IL) mediation during this process is necessary for lymphocyte activation, proliferation and differentiation, and activation of further inflammatory responses [72]. EBV miRNAs can target the inhibition of IL or IL receptor expression, block IL-mediated lymphocyte differentiation and immune activation. For example, IL1R1 is a receptor for IL-1. When EBV virus invades, IL-1 signaling pathway plays an important role in triggering an inflammatory response and host innate immune response [73]. Skinner, C.M., et al. that EBV miR-BHRF2-5p can directly target The IL-1 receptor (L1R1), the activation of IL-L $\beta$-induced NF-kB, and inhibits activation of host lymphocytes and immune responses [74]. IL-6 can promote the proliferation of B cells and T cells, stimulate the activation of CTL cells, and participate in the inflammatory response [75], while EBV miR-BART6-3p can target IL-6 receptors and inhibit lymphocyte immune activation [76]. IL-12 promotes $\mathrm{T}$ cell proliferation, induces Th0 cells differentiation into Th1 cells, and activates cytotoxicity of CTL cells and NK cells [77], while EBV miR-BART1, EBV miR-BART2, EBV miR-BART22 and EBV miR-BHRF1-2 significantly inhibited the expression and release of IL-12 [56]. Similarly, EBV miR-BART15-3p directly targets the 3'UTR of inflammasome NLRP3 mRNA, inhibits NLRP3 expression, and indirectly inhibits IL-1 $\beta$ expression, thereby attenuating host-induced immune responses [78].

\section{Interferon expression and release}

When the host is infected by EBV, it not only expresses and releases IL to resist virus infection, but also expresses Interferons (IFNs) and releases. IFNs can up-regulate the activity of various immune cells, such as macrophages, dendritic cells (DC), B lymphocytes, $\mathrm{T}$ lymphocytes and NK cells, so as to exert anti-viral and anti-tumor effects [79]. EBV-miRNAs were found to target and regulate the genes involved in the IFNs signaling pathway, including T-bet, CXCL11, CLEC2D, RIG-I, and CREBBP, to inhibit the expression and release of INFs, and inhibiting the activation of immune responses and immunotoxicity.

The $\mathrm{T}$ cell-mediated transcriptional regulator T-bet is a transcriptional activator of IFN- $\gamma$ and regulates the production of IL-2 and Th2 cytokines [80]. Tokunaga, R., et al. has found that EBV miR-BART20-5p directly targets the inhibition of T-bet expression in invasive EBV+ nasal NK/T-cell lymphoma, and EBV miR-BART8 can indirectly block IFN- $\gamma /$ STAT1 pathway, can inhibit IFN- $\gamma$ activation of the immune response. CXCL11 is an interferoninduced chemokine, and its corresponding chemokine receptor CXCR3 is expressed on the surface of NK cells and Th1 cells. When EBV invades host cells, CXCL11 on the host cells can specifically bind to CXCR3, thereby recruiting NK Cells and Th1 cells, and activate them [81]. Xia, $\mathrm{T}$ et al. predicted that EBV miR-BHRF1-3 directly targets CXCL11, and inhibits the expression of endogenous EBV miR-BHRF1-3 in EBV-positive B cells, which can significantly increase CXCL11 mRNA levels, indicating EBV miR-BHRF1-3 directly or indirectly regulate CXCL11 expression, inhibit the recruitment and activation of NK cells and Th1 cells $[82,83]$. CLEC2D is expressed on the surface of B lymphocytes, promotes the expression and release of IFN- $\gamma$ by interacting with CD161 molecules on lymphocytes, and activates NK cells and T cells 
[84], while EBV miR-BART1-3p and EBV miR-BART3-3p can target the 3'UTR of CLEC2D mRNA, inhibit the expression and release of IFN- $\gamma$, and block the activation of NK cells and T cells [54]. The retinoic acid-inducible gene-I (RIG-I) recognizes EBV nucleic acid in the cytoplasm and induces the expression and secretion of IFNs, activates NK cells and $T$ cells [85], while EBV miR-6-3p can bind to the 3'UTR of RIG-I mRNA inhibits the expression and release of IFNs, and inhibits the immune activation of lymphocytes [86]. The CREBBP gene is a coactivator of transcription of the type I IFN signaling pathway, and activates the expression and release of IFNs [87]. It was found that EBV-miR-BART16 can target CREBBP and block the expression and release of virus-induced type I IFNs, suppress immune activation and response and assist host immune escape $[88,89]$.

\section{Exosomes assist tumor cell immune escape}

In addition to directly acting on genes in host cells, EBV-miRNAs synthesized by EBV can also secrete exosomes through host cells, and use exosomes as vectors to transfer EBV-miRNAs from host cells to adjacent or distant cells. It regulates the expression of genes in other cells, exerts biological functions, and cooperates to achieve immune evasion [90]. It has found that in EBV-related tumors (such as nasopharyngeal carcinoma, Burkitt's lymphoma, Hodgkin's lymphoma, NK/T-cell lymphoma, gastric cancer), exosomes carrying EBV-miRNAs can promote tumor cell immune escape and promote progression of the tumor [91]. In EBV-associated tumor tissues, tumor infiltrating lymphocytes (TILs) can inhale EBV-miR-BARTs by endocytosis, and these EBV miRNAs can significantly reduce the immune recognition and cytotoxicity of TILs. For example, EBV-miR-BART3 inhaled can target the 3'UTR of IPO7 mRNA in TILs, inhibit the expression of IPO7 in TILs, and reduce its specific cytotoxicity against tumor cells, thus assisting tumor cell immune escape [68].

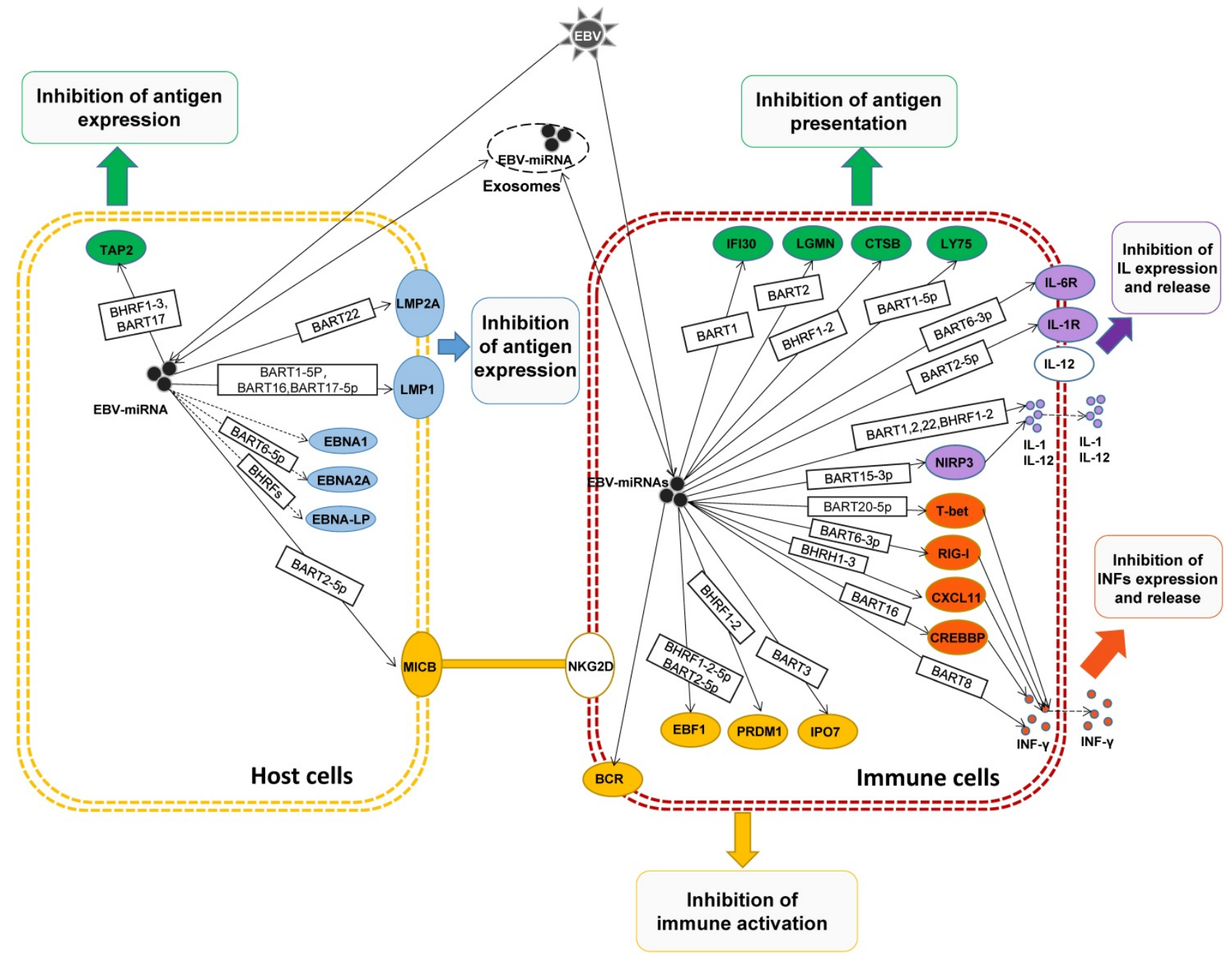

Figure 3. EBV miRNA regulate host immune responses 
In general, EBV-miRNAs are delivered to immune-related cells by exocytosis release, transmission and endocytosis, regulating the expression of related genes, suppressing immune responses, synergistically promoting the immune escape of the host, and promoting tumor development.

\section{Conclusions}

To sum up, after EBV infection host cells, in the face of the host in the process of the body's immune response of attack, EBV miRNAs by adjusting the EBV antigen expression in host cells, interfere with antigen presentation, regulating effect and toxicity of lymphocyte, so as to derive a series of getting rid of host immune surveillance and immune attack strategy, to assist the host cell and EBV immune escape, achieve long-term latent EBV infection, and promote the development of host cells to immortalization and tumors. And also can use host secrete exosomes, EBV miRNAs are transferred to adjacent or distant tumor cells and play a corresponding biological function to assist the immune escape of tumor cells.

At present, there are still many biological functions and mechanisms of EBV miRNAs that are not well clear. Even the well-defined functional EBV miRNAs may have other biological functions and mechanisms that have not yet been discovered, and there is still much work to be done. More or comprehensive disclosure of the targets and mechanisms of EBV miRNAs will play an important role in elucidating the mechanisms between EBV and the host immune system, EBV and host cell immortalization. It may provide an alternative immunotherapy approach for EBV-associated tumors as a potential target.

\section{Acknowledgments}

This work was supported by grants from the Shenzhen Nanshan District Science and Technology Innovation Bureau Education (Health) science and technology funding project (2018005).

\section{Competing Interests}

The authors have declared that no competing interest exists.

\section{References}

1. Nowalk A, Green M. Epstein-Barr Virus. Microbiol Spectr. 2016; 4.

2. Epstein MA, Achong BG, Barr YM. Virus Particles in Cultured Lymphoblasts from Burkitt's Lymphoma. Lancet. 1964; 1: 702-3.

3. Morales-Sanchez A, Fuentes-Panana EM. Epstein-Barr Virus-associated Gastric Cancer and Potential Mechanisms of Oncogenesis. Curr Cancer Drug Targets. 2017; 17: 534-54.

4. Pattle SB, Farrell PJ. The role of Epstein-Barr virus in cancer. Expert Opin Biol Ther. 2006; 6: 1193-205.

5. Shannon-Lowe C, Rickinson AB, Bell AI. Epstein-Barr virus-associated lymphomas. Philos Trans R Soc Lond B Biol Sci. 2017; 372.
6. Yachie A, Kanegane H, Kasahara Y. Epstein-Barr virus-associated $\mathrm{T}$-/natural killer cell lymphoproliferative diseases. Semin Hematol. 2003; 40: $124-32$.

7. Kiraz Y, Adan A, Kartal Yandim M, Baran Y. Major apoptotic mechanisms and genes involved in apoptosis. Tumour Biol. 2016; 37: 8471-86.

8. de-The G, Day NE, Geser A, Lavoue MF, Ho JH, Simons MJ, et al. Sero-epidemiology of the Epstein-Barr virus: preliminary analysis of an international study - a review. IARC Sci Publ. 1975: 3-16.

9. Crombie JL, LaCasce AS. Epstein Barr Virus Associated B-Cell Lymphomas and Iatrogenic Lymphoproliferative Disorders. Front Oncol. 2019; 9: 109.

10. Cohen JI. Epstein-Barr virus infection. N Engl J Med. 2000; 343: 481-92.

11. Ressing ME, van Gent M, Gram AM, Hooykaas MJ, Piersma SJ, Wiertz EJ. Immune Evasion by Epstein-Barr Virus. Curr Top Microbiol Immunol. 2015; 391: 355-81.

12. Moss DJ, Lutzky VP. EBV-Specific Immune Response: Early Research and Personal Reminiscences. Curr Top Microbiol Immunol. 2015; 390: 23-42.

13. Chiu YF, Sugden B. Epstein-Barr Virus: The Path from Latent to Productive Infection. Annu Rev Virol. 2016; 3: 359-72.

14. Kanda T. EBV-Encoded Latent Genes. Adv Exp Med Biol. 2018; 1045: 377-94.

15. Babcock GJ, Decker LL, Volk M, Thorley-Lawson DA. EBV persistence in memory B cells in vivo. Immunity. 1998; 9: 395-404.

16. Gravelle P, Pericart S, Tosolini M, Fabiani B, Coppo P, Amara N, et al. EBV infection determines the immune hallmarks of plasmablastic lymphoma. Oncoimmunology. 2018; 7: e1486950.

17. Navari M, Etebari M, De Falco G, Ambrosio MR, Gibellini D, Leoncini L, et al. The presence of Epstein-Barr virus significantly impacts the transcriptional profile in immunodeficiency-associated Burkitt lymphoma. Front Microbiol. 2015; 6: 556.

18. Chiang AK, Tao Q, Srivastava G, Ho FC. Nasal NK- and T-cell lymphomas share the same type of Epstein-Barr virus latency as nasopharyngeal carcinoma and Hodgkin's disease. Int J Cancer. 1996; 68: 285-90.

19. Sakamoto K, Sekizuka T, Uehara T, Hishima T, Mine S, Fukumoto H, et al. Next-generation sequencing of miRNAs in clinical samples of Epstein-Barr virus-associated B-cell lymphomas. Cancer Med. 2017; 6: 605-18

20. Gion Y, Iwaki N, Takata K, Takeuchi M, Nishida K, Orita Y, et al. Clinicopathological analysis of methotrexate-associated lymphoproliferative disorders: Comparison of diffuse large B-cell lymphoma and classical Hodgkin lymphoma types. Cancer Sci. 2017; 108: $1271-80$.

21. Tsao SW, Tsang CM, Lo KW. Epstein-Barr virus infection and nasopharyngeal carcinoma. Philos Trans R Soc Lond B Biol Sci. 2017; 372.

22. Castillo JJ, Beltran BE, Miranda RN, Paydas S, Winer ES, Butera JN. Epstein-barr virus-positive diffuse large B-cell lymphoma of the elderly: what we know so far. Oncologist. 2011; 16: 87-96.

23. Bansal D, Singh N, Agrawal N, Mehta A. Plasmablastic Lymphoma Versus EBV-Positive Myeloma. Indian J Hematol Blood Transfus. 2019; 35: 567-9.

24. Ambrosio MR, Mundo L, Gazaneo S, Picciolini M, Vara PS, Sayed S, et al. MicroRNAs sequencing unveils distinct molecular subgroups of plasmablastic lymphoma. Oncotarget. 2017; 8: 107356-73.

25. Meer S, Perner Y, McAlpine ED, Willem P. Extraoral plasmablastic lymphomas in a high human immunodeficiency virus endemic area. Histopathology. 2020; 76: 212-21.

26. Miao L, Guo N, Feng Y, Rao H, Wang F, Huang Q, et al. High incidence of MYC rearrangement in human immunodeficiency virus-positive plasmablastic lymphoma. Histopathology. 2020; 76: 201-11.

27. Rodrigues-Fernandes CI, de Souza LL, Santos-Costa SFD, Silva AMB, Pontes HAR, Lopes MA, et al. Clinicopathological analysis of oral plasmablastic lymphoma: A systematic review. J Oral Pathol Med. 2018; 47: 915-22.

28. Laurent C, Fabiani B, Do C, Tchernonog E, Cartron G, Gravelle P, et al. Immune-checkpoint expression in Epstein-Barr virus positive and negative plasmablastic lymphoma: a clinical and pathological study in 82 patients. Haematologica. 2016; 101: 976-84.

29. Pfeffer S, Zavolan M, Grasser FA, Chien M, Russo JJ, Ju J, et al. Identification of virus-encoded microRNAs. Science. 2004; 304: 734-6.

30. Pratt ZL, Kuzembayeva M, Sengupta S, Sugden B. The microRNAs of Epstein-Barr Virus are expressed at dramatically differing levels among cell lines. Virology. 2009; 386: 387-97.

31. Skalsky RL, Cullen BR. EBV Noncoding RNAs. Curr Top Microbiol Immunol. 2015; 391: 181-217. 
32. Hartung A, Makarewicz O, Egerer R, Karrasch M, Klink A, Sauerbrei A, et al. EBV miRNA expression profiles in different infection stages: A prospective cohort study. PLoS One. 2019; 14: e0212027.

33. Skalsky RL. Analysis of Viral and Cellular MicroRNAs in EBV-Infected Cells. Methods Mol Biol. 2017; 1532: 133-46.

34. Robertson ES, Tomkinson B, Kieff E. An Epstein-Barr virus with a 58-kilobase-pair deletion that includes BARF0 transforms B lymphocytes in vitro. J Virol. 1994; 68: 1449-58.

35. Ambros V, Bartel B, Bartel DP, Burge CB, Carrington JC, Chen X, et al. A uniform system for microRNA annotation. RNA. 2003; 9: 277-9.

36. Skalsky RL, Cullen BR. Viruses, microRNAs, and host interactions. Annu Rev Microbiol. 2010; 64: 123-41.

37. Dong $\mathrm{M}$, Chen JN, Huang JT, Gong LP, Shao CK. The roles of EBV-encoded microRNAs in EBV-associated tumors. Crit Rev Oncol Hematol. 2019; 135: 30-8.

38. Scott RS. Epstein-Barr virus: a master epigenetic manipulator. Curr Opin Virol. 2017; 26: 74-80

39. Worth AJ, Houldcroft CJ, Booth C. Severe Epstein-Barr virus infection in primary immunodeficiency and the normal host. Br J Haematol. 2016; 175: 559-76.

40. Cen O, Longnecker R. Latent Membrane Protein 2 (LMP2). Curr Top Microbiol Immunol. 2015; 391: 151-80.

41. Lung RW, Tong JH, Sung YM, Leung PS, Ng DC, Chau SL, et al. Modulation of LMP2A expression by a newly identified Epstein-Barr virus-encoded microRNA miR-BART22. Neoplasia. 2009; 11: 1174-84.

42. Wang LW, Jiang S, Gewurz BE. Epstein-Barr Virus LMP1-Mediated Oncogenicity. J Virol. 2017; 91.

43. Albanese M, Tagawa T, Buschle A, Hammerschmidt W. MicroRNAs of Epstein-Barr Virus Control Innate and Adaptive Antiviral Immunity. J Virol. 2017; 91.

44. Ramakrishnan R, Donahue H, Garcia D, Tan J, Shimizu N, Rice AP, et al. Epstein-Barr virus BART9 miRNA modulates LMP1 levels and affects growth rate of nasal NK T cell lymphomas. PLoS One. 2011; 6: e27271.

45. Zhang Y, Zhang W, Liu W, Liu H, Zhang Y, Luo B. Epstein-Barr virus miRNA-BART16 modulates cell proliferation by targeting LMP1. Virus Res. 2018; 256: 38-44.

46. Lo AK, To KF, Lo KW, Lung RW, Hui JW, Liao G, et al. Modulation of LMP1 protein expression by EBV-encoded microRNAs. Proc Natl Acad Sci U S A. 2007; 104: 16164-9.

47. Albanese M, Tagawa T, Bouvet M, Maliqi L, Lutter D, Hoser J, et al. Epstein-Barr virus microRNAs reduce immune surveillance by virus-specific CD8+ T cells. Proc Natl Acad Sci U S A. 2016; 113: E6467-E75.

48. Gordadze AV, Poston D, Ling PD. The EBNA2 polyproline region is dispensable for Epstein-Barr virus-mediated immortalization maintenance. J Virol. 2002; 76: 7349-55.

49. Poling BC, Price AM, Luftig MA, Cullen BR. The Epstein-Barr virus miR-BHRF1 microRNAs regulate viral gene expression in cis. Virology. 2017; 512: 113-23.

50. McCluskey J, Rossjohn J, Purcell AW. TAP genes and immunity. Curr Opin Immunol. 2004; 16: 651-9.

51. Lin TC, Liu TY, Hsu SM, Lin CW. Epstein-Barr virus-encoded miR-BART20-5p inhibits T-bet translation with secondary suppression of p53 in invasive nasal NK/T-cell lymphoma. Am J Pathol. 2013; 182: 1865-75.

52. Weiss A, Littman DR. Signal transduction by lymphocyte antigen receptors. Cell. 1994; 76: 263-74

53. Zhao C, Liu Q, Xu S, Xiao Y, Wang W, Yang J, et al. Identification of type A spermatogonia in turbot (Scophthalmus maximus) using a new cell-surface marker of Lymphocyte antigen 75 (ly75/CD205). Theriogenology. 2018; 113: 137-45.

54. Skalsky RL, Corcoran DL, Gottwein E, Frank CL, Kang D, Hafner M, et al. The viral and cellular microRNA targetome in lymphoblastoid cell lines. PLoS Pathog. 2012; 8: e1002484.

55. Anderson HA, Roche PA. MHC class II association with lipid rafts on the antigen presenting cell surface. Biochim Biophys Acta. 2015; 1853: 775-80.

56. Tagawa T, Albanese M, Bouvet M, Moosmann A, Mautner J, Heissmeyer $\mathrm{V}$, et al. Epstein-Barr viral miRNAs inhibit antiviral CD4+ $\mathrm{T}$ cell responses targeting IL-12 and peptide processing. J Exp Med. 2016; 213: 2065-80.

57. Wang F. Nonhuman primate models for Epstein-Barr virus infection. Curr Opin Virol. 2013; 3: 233-7.

58. Apcher S, Fahraeus R, Manoury B. Epstein-Barr virus: exploiting the immune system by interfering with defective ribosomal products. Microbes Infect. 2004; 6: 1212-8.

59. Nachmani D, Stern-Ginossar N, Sarid R, Mandelboim O, Diverse herpesvirus microRNAs target the stress-induced immune ligand MICB to escape recognition by natural killer cells. Cell Host Microbe. 2009; 5: 376-85.

60. Gyory I, Boller S, Nechanitzky R, Mandel E, Pott S, Liu E, et al Transcription factor Ebf1 regulates differentiation stage-specific signaling, proliferation, and survival of B cells. Genes Dev. 2012; 26: 668-82.

61. Maier H, Hagman J. Roles of EBF and Pax-5 in B lineage commitment and development. Semin Immunol. 2002; 14: 415-22.

62. Ross N, Gandhi MK, Nourse JP. The Epstein-Barr virus microRNA BART11-5p targets the early B-cell transcription factor EBF1. Am J Blood Res. 2013; 3: 210-24

63. Boi M, Zucca E, Inghirami G, Bertoni F. PRDM1/BLIMP1: a tumor suppressor gene in B and T cell lymphomas. Leuk Lymphoma. 2015; 56: 1223-8.

64. Ghraichy M, Galson JD, Kelly DF, Truck J. B-cell receptor repertoire sequencing in patients with primary immunodeficiency: a review. Immunology. 2018; 153: 145-60.

65. Chen Y, Fachko D, Ivanov NS, Skinner CM, Skalsky RL. Epstein-Barr virus microRNAs regulate $B$ cell receptor signal transduction and lytic reactivation. PLoS Pathog. 2019; 15: e1007535.

66. Ma I, Nie K, Redmond D, Liu Y, Elemento O, Knowles DM, et al. EBV-miR-BHRF1-2 targets PRDM1/Blimp1: potential role in EBV lymphomagenesis. Leukemia. 2016; 30: 594-604.

67. Szczyrba J, Nolte E, Hart M, Doll C, Wach S, Taubert H, et al. Identification of ZNF217, hnRNP-K, VEGF-A and IPO7 as targets for microRNAs that are downregulated in prostate carcinoma. Int J Cancer. 2013; 132: 775-84

68. Dolken L, Malterer G, Erhard F, Kothe S, Friedel CC, Suffert G, et al. Systematic analysis of viral and cellular microRNA targets in cells latently infected with human gamma-herpesviruses by RISC immunoprecipitation assay. Cell Host Microbe. 2010; 7: 324-34.

69. Hachmann J, Salvesen GS. The Paracaspase MALT1. Biochimie. 2016; 122: 324-38.

70. Uehata T, Iwasaki H, Vandenbon A, Matsushita K, Hernandez-Cuellar E, Kuniyoshi K, et al. Malt1-induced cleavage of regnase-1 in CD4(+) helper T cells regulates immune activation. Cell. 2013; 153: 1036-49.

71. Wang YF, He DD, Liang HW, Yang D, Yue H, Zhang XM, et al. The identification of up-regulated ebv-miR-BHRF1-2-5p targeting MALT1 and ebv-miR-BHRF1-3 in the circulation of patients with multiple sclerosis. Clin Exp Immunol. 2017; 189: 120-6

72. Huang $\mathrm{Y}, \mathrm{Li}$ D, Qin DY, Gou HF, Wei W, Wang YS, et al. Interleukin-armed chimeric antigen receptor-modified $\mathrm{T}$ cells for cancer immunotherapy. Gene Ther. 2018; 25: 192-7.

73. Dinarello CA. Overview of the IL-1 family in innate inflammation and acquired immunity. Immunol Rev. 2018; 281: 8-27.

74. Skinner CM, Ivanov NS, Barr SA, Chen Y, Skalsky RL. An Epstein-Barr Virus MicroRNA Blocks Interleukin-1 (IL-1) Signaling by Targeting IL-1 Receptor 1. J Virol. 2017; 91.

75. Rose-John S. Interleukin-6 Family Cytokines. Cold Spring Harb Perspect Biol. 2018; 10

76. Ambrosio MR, Navari M, Di Lisio L, Leon EA, Onnis A, Gazaneo S, et al. The Epstein Barr-encoded BART-6-3p microRNA affects regulation of cell growth and immuno response in Burkitt lymphoma. Infect Agent Cancer. 2014; 9: 12 .

77. Guo Y, Cao W, Zhu Y. Immunoregulatory Functions of the IL-12 Family of Cytokines in Antiviral Systems. Viruses. 2019; 11.

78. Haneklaus M, Gerlic M, Kurowska-Stolarska M, Rainey AA, Pich D, McInnes IB, et al. Cutting edge: miR-223 and EBV miR-BART15 regulate the NLRP3 inflammasome and IL-1beta production. J Immunol. 2012; 189: 3795-9.

79. Dunn GP, Koebel CM, Schreiber RD. Interferons, immunity and cancer immunoediting. Nat Rev Immunol. 2006; 6: 836-48.

80. Mohamed R, Lord GM. T-bet as a key regulator of mucosal immunity. Immunology. 2016; 147: 367-76

81. Tokunaga R, Zhang W, Naseem M, Puccini A, Berger MD, Soni S, et al. CXCL9, CXCL10, CXCL11/CXCR3 axis for immune activation - A target for novel cancer therapy. Cancer Treat Rev. 2018; 63: 40-7.

82. Egesten A, Eliasson M, Olin AI, Erjefalt JS, Bjartell A, Sangfelt P, et al. The proinflammatory CXC-chemokines GRO-alpha/CXCL1 and MIG/CXCL9 are concomitantly expressed in ulcerative colitis and decrease during treatment with topical corticosteroids. Int J Colorectal Dis. 2007; 22: 1421-7.

83. Xia T, O'Hara A, Araujo I, Barreto J, Carvalho E, Sapucaia JB, et al. EBV microRNAs in primary lymphomas and targeting of CXCL-11 by ebv-mir-BHRF1-3. Cancer Res. 2008; 68: 1436-42.

84. Mathew SO, Chaudhary P, Powers SB, Vishwanatha JK, Mathew PA. Overexpression of LLT1 (OCIL, CLEC2D) on prostate cancer cells inhibits NK cell-mediated killing through LLT1-NKRP1A (CD161) interaction. Oncotarget. 2016; 7: 68650-61. 
85. Loo YM, Gale M, Jr. Immune signaling by RIG-I-like receptors. Immunity. 2011; 34: 680-92.

86. Lu Y, Qin Z, Wang J, Zheng X, Lu J, Zhang X, et al. Epstein-Barr Virus miR-BART6-3p Inhibits the RIG-I Pathway. J Innate Immun. 2017; 9: 574-86.

87. Jiang Y, Ortega-Molina A, Geng H, Ying HY, Hatzi K, Parsa S, et al. CREBBP Inactivation Promotes the Development of HDAC3-Dependent Lymphomas. Cancer Discov. 2017; 7: 38-53.

88. Hooykaas MJG, van Gent M, Soppe JA, Kruse E, Boer IGJ, van Leenen D, et al. EBV MicroRNA BART16 Suppresses Type I IFN Signaling. J Immunol. 2017; 198: 4062-73.

89. Tang X, Gao JS, Guan YJ, McLane KE, Yuan ZL, Ramratnam B, et al. Acetylation-dependent signal transduction for type I interferon receptor. Cell. 2007; 131: 93-105.

90. Khan G, Philip PS. Functional Analysis of Exosomes Derived from EBV-Infected Cells. Methods Mol Biol. 2017; 1532: 159-67.

91. Teow SY, Liew K, Khoo AS, Peh SC. Pathogenic Role of Exosomes in Epstein-Barr Virus (EBV)-Associated Cancers. Int J Biol Sci. 2017; 13: 1276-86. 